diferencias significativas en la esperanza de vida, el $\mathrm{PIB} / \mathrm{c}$ y la desigualdad de los ingresos en los dos subgrupos de países de cada una de las cuatro categorías. Las mayores diferencias en cuanto al PIB/c y a la desigualdad de los ingresos se observaron entre los países miembros de la OCDE y los que no eran miembros de esa organización, mientras que la mayor diferencia en cuanto a la esperanza de vida se encontró entre los países considerados periféricos y los no periféricos. El análisis combinado de los datos demostró que los ingresos tienen un efecto directo significativo sobre la esperanza de vida y confirmó el efecto adverso de la desigualdad de los ingresos sobre la esperanza de vida.

No se encontraron diferencias significativas en la esperanza de vida, el PIB/c y la desigualdad de los ingresos ni entre los países de ingresos altos y bajos ni entre los países pertenecientes o no al núcleo comercial.

Estos resultados permiten rechazar la hipótesis de que las diferencias en los ingresos de los países reflejan mejor su estratificación en ricos y pobres y que los efectos negativos de la desigualdad en los ingresos son mayores en los países de ingresos altos. Por el contrario, demuestran que a partir de los patrones comerciales se logra una mejor estratificación de los países y que la desigualdad en los ingresos tiene un efecto adverso sobre la esperanza de vida en países de la periferia. (Moore $S$. Peripherality, income inequality, and life expectancy: revisiting the income inequality hypothesis. Int J Epidemiol. 2006;35(3):623-32.)

\section{Problemas de salud de los adultos mayores en una población de la frontera entre México y los Estados Unidos}

La frontera entre los Estados Unidos de América y México es un área excepcional desde muchos puntos de vista. Además de considerarse una de las regiones más dinámicas del mundo, el frecuente cruce de la frontera influye en la cultura y el estilo de vida de las poblaciones asentadas en su entono. El rápido aumento de la población en la frontera afecta a la infraestructura de la atención sanitaria y representa un reto para los profesionales de la salud y las autoridades encargadas de eliminar las desigualdades de salud y de ofrecer una atención ajustada a las características culturales de esa población, especialmente de los adultos mayores.

En este estudio se analizan los problemas de este grupo de la población en un condado rural de Arizona, Estados Unidos, fronterizo con México. Mediante entrevistas a 29 informantes clave - proveedores de servicios sociales y de salud y líderes comunitarios- y con datos de los archivos públicos se analizaron: 1) el estado de salud de los adultos mayores, 2) sus necesidades de atención sanitaria y su patrón de utilización, 3) la capacidad de la comunidad de satisfacer las necesidades de los adultos mayores y las deficiencias de la atención, 4) la influencia de la cultura en las creencias y prácticas relacionadas con la salud y 5) las necesidades educacionales de los profesionales de la salud relacionadas con el envejecimiento y el cuidado de los adultos mayores.

Los resultados de este estudio indicaron que existen desigualdades que afectan a la salud de los adultos mayores en la zona fronteriza. El principal problema que impide eliminar esas desigualdades es el insuficiente acceso de los adultos mayores a la atención sanitaria en esta región. Esto no solo se debió a problemas financieros, sino también a la insuficiente infraestructura de la comunidad, a las condiciones geográficas, demográficas y socioeconómicas, al poco conocimiento de esta población acerca de la salud, a las particularidades de la cultura dominante, al desconocimiento de la población acerca del envejecimiento y de los problemas específicos relacionados con cruzar la frontera. La diversidad de temas relacionados con la salud de los adultos mayores en esta zona indica que las causas de esas desigualdades son multifactoriales.

El impacto de las diferencias culturales en los conceptos y prácticas relacionados con la salud se manifestó tanto en los pacientes como en los proveedores de los servicios de salud. Por ello, el reto para los proveedores de asistencia sanitaria consiste en desarrollar un sistema competente desde el punto de vista cultural y conocer mejor las particularidades socioculturales de esta comunidad y de sus adultos mayores.

Los adultos mayores que viven en la zona fronteriza constituyen una población especialmente vulnerable. A pesar de que este grupo de la población víctima de la desigualdad ha crecido, el enfoque tradicional de los sistemas de salud ha contribuido a que se desatiendan sus necesidades de salud. La comunidad, los encargados de establecer las políticas y las agencias y proveedores de servicios sociales y de salud deben informarse mejor acerca de los problemas que aquejan a los adultos mayores y preparar adecuadamente al personal para brindar una atención de salud de calidad y sensible al entorno cultural. En este trabajo se propone un marco de acción para desarrollar programas educativos y proyectos de investigación dirigidos a los profesionales de salud encargados de atender a los adultos mayores que viven en esta zona fronteriza. (Guo G, Phillips L. Key informants' perceptions of health care for elders at the U.S.-Mexico border. Public Health Nurs. 2006; 23(3):224.) 